\title{
Efficacy of the Phase Images in Fourier Analysis Using Gated Cardiac POOL-SPECT for Determining the Indication for Cardiac Resynchronization Therapy
}

\author{
Toshihiro Muramatsu, MD; Kazuo Matsumoto, MD; Shigeyuki Nishimura, MD
}

\begin{abstract}
Background Although cardiac resynchronization therapy (CRT) improves quality of life and survival for patients with heart failure, exact methods to estimate the effect of cardiac asynchrony have not yet been defined. Methods and Results Initially, to examine whether the phase analysis images in the Fourier analysis using gated cardiac pool single photon emission computed tomography (POOL-SPECT) could be used to evaluate cardiac asynchrony, 19 consecutive patients with dilated cardiomyopathy were studied. Interventricular asynchrony was defined by whether the peak of the picture elements of the right ventricle in the phase histogram fitted that of the left ventricle and intraventricular asynchrony by whether the phase image was described homogenously or not. The patients with both inter- and intraventricular asynchrony had significant deterioration in both left ventricular ejection fraction $(\mathrm{p}<0.01)$ and New York Heart Association functional class $(\mathrm{p}<0.01)$. To evaluate the efficacy of these phase images for CRT setting, 7 patients were tested before and after CRT. During a 3.9 \pm 3.6 month follow-up period, all patients had an improvement in their condition, and the inter- and intraventricular asynchrony significantly improved after CRT. The degrees of the inter- and intraventricular asynchrony were related to the degree of cardiac depression pre CRT.

Conclusion These results have shown that the phase images from POOL-SPECT are useful for assessing the effect of CRT in patients with heart failure, which suggests that it may provide information about the indication for CRT. (Circ J 2005; 69: 1521-1526)
\end{abstract}

Key Words: Advanced heart failure; Cardiac resynchronization therapy; Gated cardiac pool SPECT; Phase analysis image

I ntraventricular conduction delay is clearly found in some patients with heart failure and these conduction abnormalities might be related to a delay in the onset of right or left ventricular (LV) contraction. Inefficient cardiac contraction caused by such mechanical asynchrony may be partly responsible for further impairment of LV function. Cardiac resynchronization therapy (CRT) restores optimum synchrony of ventricular contraction. A metaanalysis of 4 randomized controlled trials showed that the morbidity of progressive heart failure decreased by $51 \%$ after CRT, and the admission rate was decreased by $29 \% !^{1-3}$ The aim of the present study was to evaluate ventricular asynchrony by the phase images obtained by Fourier analysis using gated cardiac pool emission computed tomography, and to evaluate whether these images can be used to determine the indication for CRT.

\section{Methods}

\section{Study Patients}

Initially, we examined the efficacy of phase image from pool single photon emission computed tomography (POOLSPECT) for evaluating heart failure in 19 patients with idiopathic dilated cardiomyopathy (DCM; 16 men, 3 women;

(Received June 10, 2005; revised manuscript received September 22, 2005; accepted October 4, 2005)

Division of Cardiology, Saitama Medical School, Saitama, Japan

Mailing address: Toshihiro Muramatsu, MD, Division of Cardiology, Saitama Medical School, 38 Moroyama-machi, Iruma-gun, Saitama

350-0495, Japan. E-mail: toshi_m@saitama-med.ac.jp

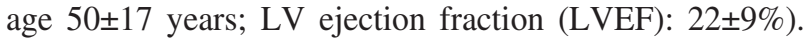
Of these, 3 patients were New York Heart Association (NYHA) functional class I, 7 were class II, and 9 were class III. Our definition of wide QRS was $>120 \mathrm{~ms}$ in duration and 7 patients fitted the definition (Table 1). This study was performed consecutively and continuously between November 2001 and December 2004. All patients were treated with angiotensin-converting enzyme inhibitors, diuretics and $\beta$-blockers during follow-up. In the next stage of the study, to evaluate the efficacy of the phase images for CRT setting, the 7 patients with advanced heart failure and wide QRS complex who underwent CRT were studied (5 men, 2 women; age $63 \pm 16$ years; DCM 6 , ischemic cardiomyopathy 1; 6 NYHA III, 1 class IIM). Phase imaging using POOL-SPECT was performed before and within 11 months (3.8 \pm 3.6 month) after CRT between October 2002 and October 2004.

\section{POOL-SPECT}

POOL-SPECT was performed using technetium-99m $(99 \mathrm{mTc})$ red blood cells with an in vivo labeling method. $99 \mathrm{mTc}$ pertechnetate $(740 \mathrm{MBq})$ was injected intravenously. POOL-SPECT imaging used a Prism 3000XP with a 3headed single photon emission computed tomography (SPECT) system that had low-energy, high resolution, parallel-hole collimators. A total of 72 projection images obtained over 360 degrees every 5 degrees of rotation were acquired in $64 \times 64$ matrices on a magnetic disk with 16 frames/cardiac cycle. The data acquisition time was $40 \mathrm{~s}$ for each projection. Reconstructed images were made 
Table 1 Patient Characteristics

\begin{tabular}{cccccccccc}
\hline $\begin{array}{c}\text { Patient } \\
\text { no. }\end{array}$ & Sex & $\begin{array}{c}\text { Age } \\
\text { (years) }\end{array}$ & Rhythm & $\begin{array}{c}\text { QRS width } \\
(\text { ms })\end{array}$ & NYHA & LVEF & RVEF & $\begin{array}{c}\text { EDV } \\
(\text { ml })\end{array}$ & $\begin{array}{c}\text { Follow-up } \\
\text { (months) }\end{array}$ \\
\hline 1 & $M$ & 43 & $S R$ & 106 & $I$ & 47 & 35 & 111 & 24 \\
2 & $M$ & 36 & $S R$ & 120 & $I$ & 30 & 25 & 243 & 12 \\
3 & $F$ & 61 & $S R$ & 111 & III & 17 & 25 & 199 & 30 \\
4 & $M$ & 40 & $S R$ & 104 & $I$ & 30 & 38 & 200 & 30 \\
5 & $F$ & 48 & $S R$ & 131 (intravent) & II & 21 & 23 & 393 & 24 \\
6 & $M$ & 24 & $S R$ & 118 & III & 12 & 16 & 459 & 8 \\
7 & $M$ & 35 & $S R$ & 95 & III & 21 & 13 & 306 & 15 \\
8 & $M$ & 32 & $S R$ & 106 & III & 10 & 21 & 236 & 35 \\
9 & $F$ & 66 & $S R$ & 127 (intravent) & II & 30 & 24 & 196 & 12 \\
10 & $M$ & 54 & $S R$ & 98 & II & 28 & 36 & 188 & 24 \\
11 & $M$ & 76 & $S R$ & 75 & $I I$ & 27 & 40 & 222 & 12 \\
12 & $M$ & 47 & $S R$ & 127 (intravent) & III & 16 & 48 & 324 & 24 \\
13 & $M$ & 76 & $S R$ & 190 (LBBB) & III & 11 & 31 & 532 & 8 \\
14 & $M$ & 70 & $V V I$ & 160 & III & 21 & 15 & 317 & 14 \\
15 & $F$ & 65 & $S R$ & 83 & $I I$ & 30 & 21 & 155 & 23 \\
16 & $M$ & 62 & $S R$ & 213 (intravent) & III & 15 & 35 & 369 & 31 \\
17 & $M$ & 54 & $S R$ & 187 (intravent) & II & 10 & 24 & 395 & 36 \\
18 & $M$ & 34 & $S R$ & 120 & III & 23 & 34 & 330 & 8 \\
19 & $M$ & 26 & $S R$ & 107 & II & 23 & 52 & 230 & 18 \\
\hline
\end{tabular}

NYHA, New York Heart Association; $L V E F$, left ventricular ejection fraction; RVEF, right ventricular ejection fraction; EDV, enddiastolic volume; SR, sinus rhythm; LBBB, left bundle branch block; VVI, ventricle $\cdot$ ventricle $\cdot$ inhibit.

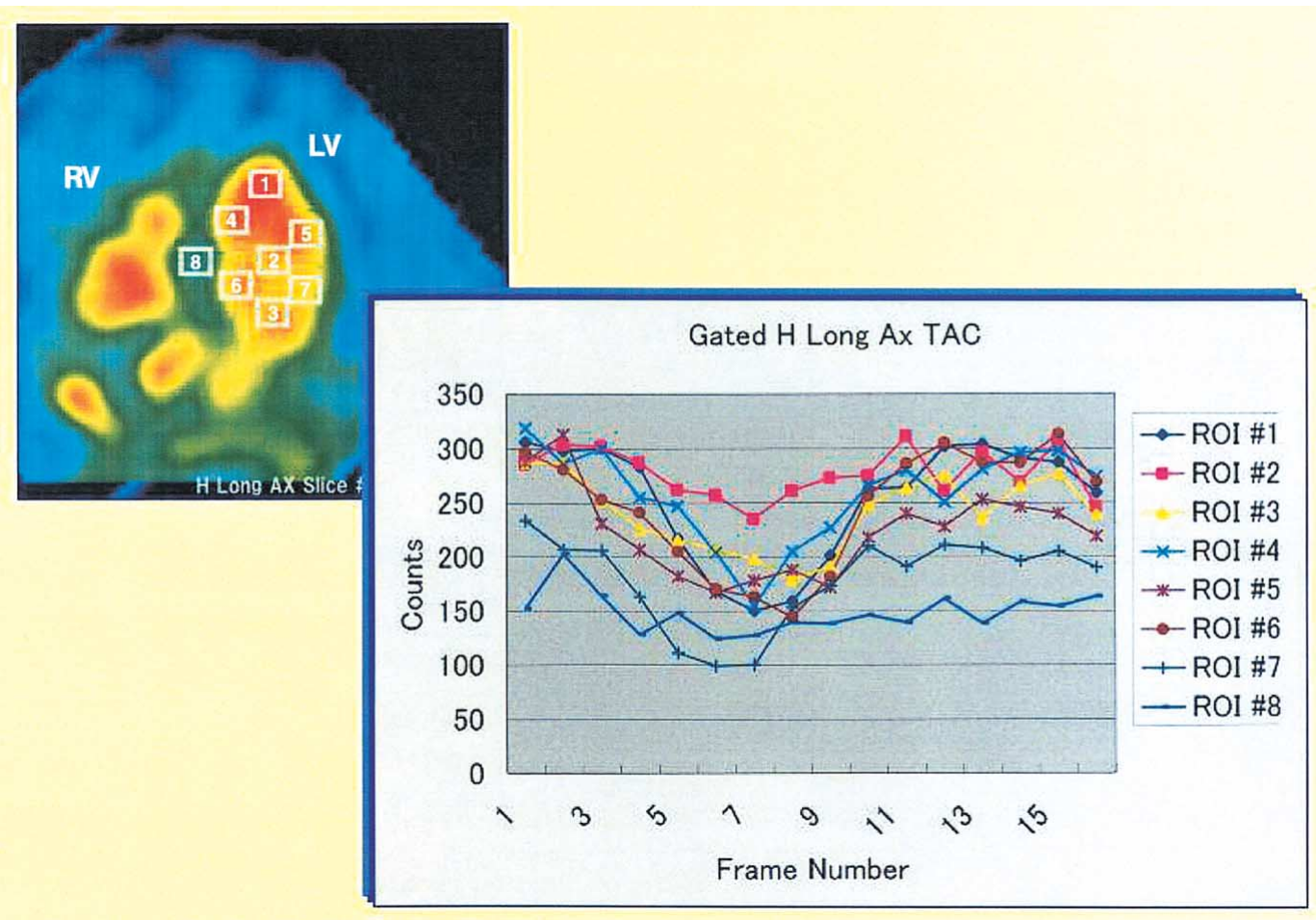

Fig 1. Links between the left ventricular segments of the horizontal long-axis view of pool single photon emission computed tomography and each time-activity curve (TAC) in the normal control patients.

up of 3 cross-sectional images such as the serial short-axis view of either side of the ventricle, the sagittal vertical long-axis view and the horizontal long-axis view. Each image was evaluated visually in cine-mode, which is the same as for quantitative gated SPECT myocardial perfusion images. The phase images are automatically produced by Prism IRIX software. Although all images were available for observation, we chose the horizontal long-axis view because it is an easily obtained view of both right and left ventricles.
Phase Analysis Images

The phase and amplitude of the fundamental wave of the discrete Fourier transform in each pixel's time-activity curve (TAC) were calculated and mapped as functional images? These phase distribution histograms described the number of pixels evaluating the end-systolic time angle for each ventricle and provided information about the contraction sequence quality. As shown in Fig 1, there is a clear difference between the TAC patterns inside and outside the cardiac cavity. Because the phase images are produced 
a

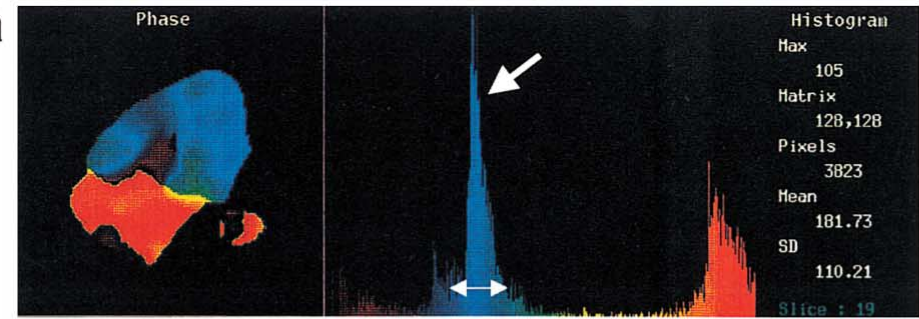

C

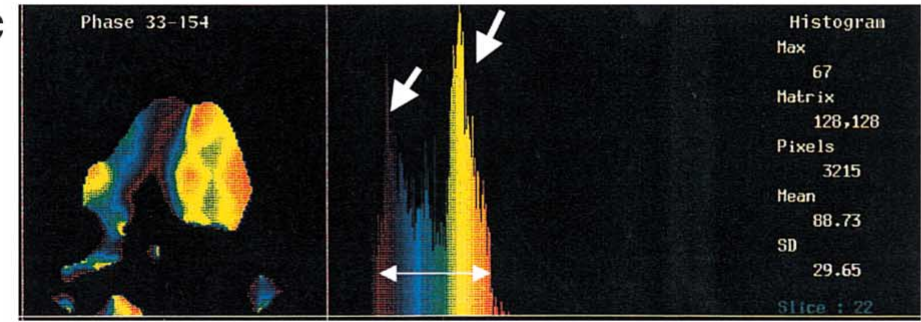

d

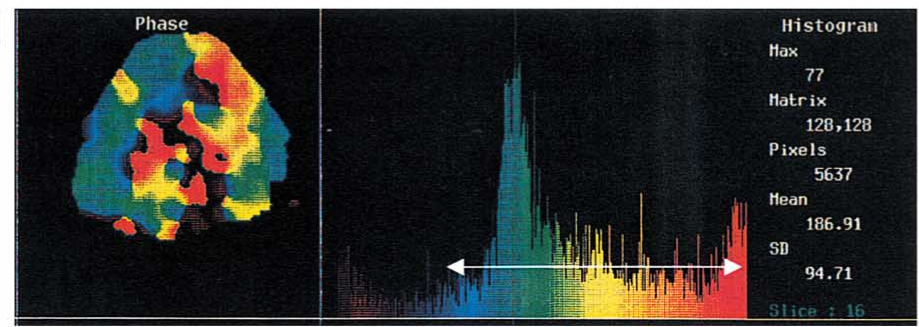

b

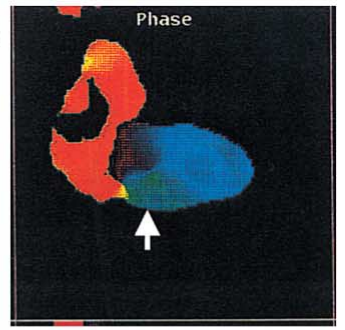

Fig 2. (a) Phase images using pool single photon emission computed tomography in a patient with a minor infarct, but normal left ventricular function. The homogeneous blue pattern in the right and left ventricles indicates well-synchronized inter- and intra-ventricular contraction. The phase histogram shows 1 peak distribution pattern, which suggests well co-ordinated contraction in both ventricles (arrows). (b) The infarcted region is shown as the darker blue region (arrow) or green as phase-delayed areas on the vertical long-axis image. (c) Phase images in a patient with a VVI pacemaker. The right ventricle is the blue region and the left ventricle is yellow. The phase histogram expresses each ingredient of the phase images graphically. The dispersion of the contraction in both ventricles has a bimodal distribution pattern (arrows). (d) Phase images in a patient with advanced heart failure. Both ventricles are heterogeneous and the phase histogram is very wide (doubleheaded arrow).

from the Fourier first harmonic transform in the TAC, the TAC of the pixels inside the cavity, which is shown in the region of interest \#2, does not provide any information concerning phase or amplitude. However, we consider that the edge's movements by tomographic count-based phase analysis reflect the phase value in the border inside the cardiac cavity.

We qualitatively evaluated the efficacy of the phase images using POOL-SPECT (Fig 2), which meant that intraventricular asynchrony was defined whether or not the phase image was described homogeneously.

We determined the inter- and intra-observer reproducibility for detecting inter- and intraventricular asynchrony in all 19 patients. The interobserver reproducibility was determined by 2 specialists in Nuclear Cardiology. The intra-observer reproducibility was determined by the original interpreter rereading the images approximately 1 month after the first reading. The inter- and intra-observer reading concordance rates were $85 \%$ and $90 \%$, respectively.

\section{CRT Setting}

An atrial lead was implanted in the right atrial appendage, and a right ventricular (RV) lead was placed at the $\mathrm{RV}$ apex. The atrioventricular delay was programmed to its optimal value using echocardiography. A LV pacing lead was inserted by a transvenous approach through the coronary sinus, and placed in the left marginal or posterior cardiac vein where the maximal blood pressure gain was obtained.

\section{Statistical Analysis}

All values are given as mean \pm standard deviation (SD). Comparisons between groups were made using Student's t-test. Linear regression analysis was carried out to correlate quantitative variables. A forward stepwise model with an entry p-value of 0.05 was used. All analysis was performed by Stat-View version5 (SAS Institute Inc, Cary, NC, USA)

\section{Results}

The correlations between the clinical findings and the presence or absence of ventricular asynchrony are summarized in Table 2. The LVEF was significantly lower in patients with both inter- and intraventricular asynchrony $(\mathrm{p}<0.01)$ and the NYHA classification was significantly higher in these patients $(\mathrm{p}<0.01)$. In the follow-up period, cardiac events occurred in 40\% (4/10) of patients with both abnormalities compared with $22 \%$ ( 2 of 9 ) of the other 2 groups and the cardiac event were more fatal. Additionally, $71 \%$ of patients with a wide QRS and $41 \%$ of patients with a narrow QRS showed both inter- and intraventricular asynchrony. Fig $2 a, b$ shows images from a patient with inferior infarction, but normal LV function (LVEF 60\%). In Fig $2 c$ the images from a patient with a VVI pacemaker 
Table 2 Correlations Between Clinical Findings and Presence or Absence of Ventricular Asynchrony According to the Phase Images Using POOL-SPECT

\begin{tabular}{|c|c|c|c|c|c|}
\hline \multirow{2}{*}{ Asynchrony } & \multicolumn{2}{|c|}{$\overline{Q Q R S \text { width }}$} & \multirow{2}{*}{$\begin{array}{c}L V E F \\
(\%)\end{array}$} & \multirow{2}{*}{ NYHA } & \multirow{2}{*}{ Cardiac event } \\
\hline & Narrow & Wide & & & \\
\hline $\begin{array}{l}\text { Interventricular }(+) \text { and } \\
\text { Intraventricular }(+)\end{array}$ & 5 & 5 & $16.6 \pm 6.0^{*}$ & $2.7 \pm 0.5 *$ & $\begin{array}{l}\text { CRT + death, CRT } \\
C R T+\text { death, LVAD }\end{array}$ \\
\hline $\begin{array}{l}\text { Interventricular }(+) \text { or } \\
\text { Intraventricular }(+)\end{array}$ & 2 & 2 & $22.8 \pm 5.4$ & $2.5 \pm 0.6$ & $\begin{array}{c}C R T \\
L V A D\end{array}$ \\
\hline $\begin{array}{l}\text { Interventricular (-) and } \\
\text { Intraventricular (-) }\end{array}$ & 5 & 0 & $32.0 \pm 8.6^{*}$ & $1.4 \pm 0.5^{*}$ & None \\
\hline
\end{tabular}

${ }^{*} p<0.01$. POOL-SPECT, pool single photon emission computed tomography; CRT, cardiac resynchronization therapy; LVAD, left ventricular assist device. See Table 1 for other abbreviations.

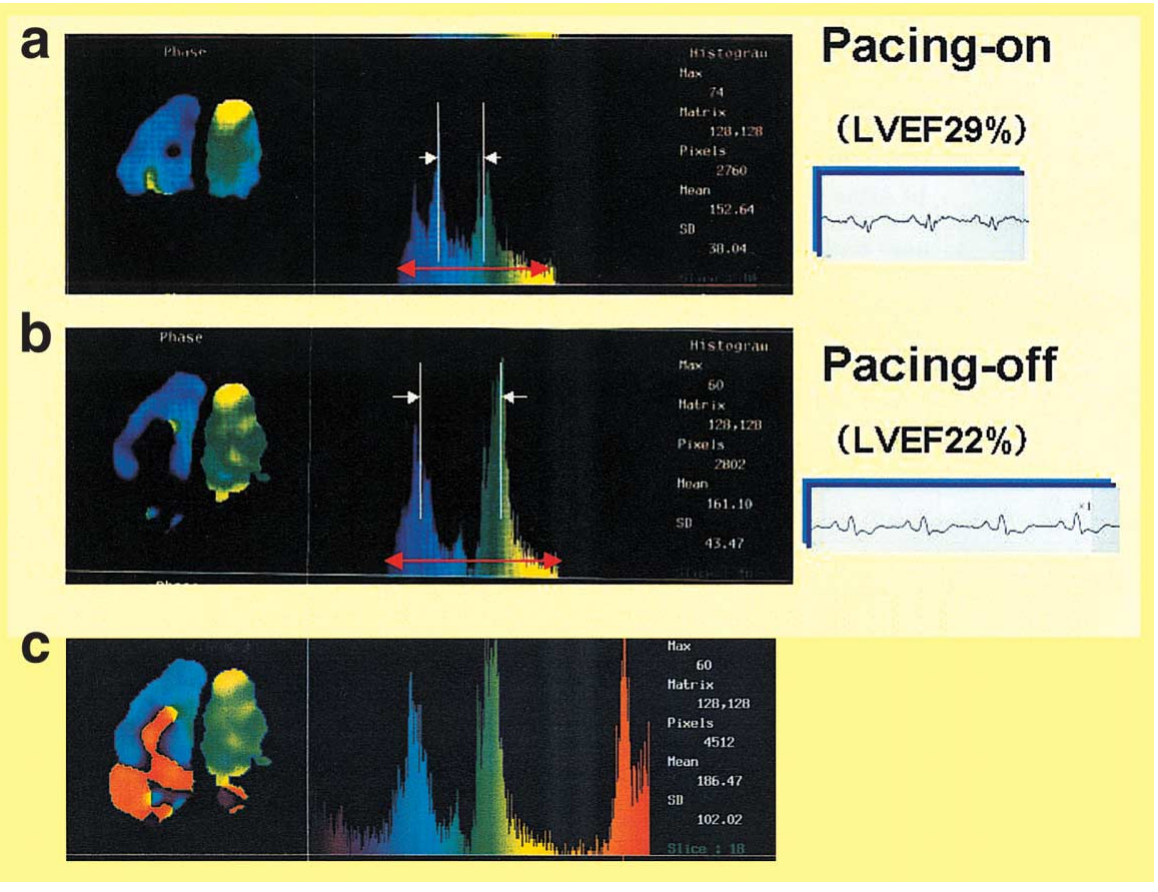

Fig 3. (a) During maintenance of resynchronization, asynchronous segments in the left ventricle became more localized. The difference in the peaks of the picture elements of both ventricles was smaller than the values for pacing-off (doubleheaded arrows). (b) After pacing was off, the phase images show the left ventricle as more multicolored. (c) Bottom image is original phase image after the pacing was off. To evaluate the only ventricular asynchrony, we removed the atrial component from the image. LVEF, left ventricular ejection fraction.

show separate contraction of the right and left ventricles, which suggests that interventricular asynchrony is defined by whether or not the peak of the picture elements of the right ventricle in the phase histogram fit those of the left ventricle. In a patient with advanced heart failure (Fig 2d) (LVEF 11\%), the phase images show that both right and left ventricles are more heterogeneous. The phase histogram is very wide and the peak of the elements of the left ventricle is not defined. These images are totally different than those in Fig 2a.

\section{Effect of CRT on Ventricular Asynchrony}

We used the phase images to understand the efficacy of CRT mechanics. Fig 3 shows images from a 32-year-old male who had undergone implantation of a LV assist device (LVAD) because of cardiac shock from DCM. Because his condition improved after the implantation, we tried CRT instead of LVAD support. During the On/Off test of CRT, as soon as pacing was off, LVEF deteriorated from $29 \%$ to $22 \%$. During maintenance of resynchronization, the asynchronous segments in the LV became more localized, suggesting that the intraventricular asynchrony was improving. We quantitatively measured the ventricular asynchrony. Intraventricular contractile asynchrony was defined as the SD of the mean phase angle for the LV and RV blood pools. The SD in this view was 38 degrees. As soon as the pacing was off, the SD extended to 43 degrees. The difference in the peaks of the picture element of both right and left ventricles was smaller during pacing-on than during pacing-off. Interventricular asynchrony was defined as the difference in the peaks of the mean phase angle between the LV and RV. During maintenance of resynchronization, the angle in this view was 39 degrees and after the pacing was off, the angle extended to 70 degrees.

Quantitative evaluation for 7 consecutive patients who underwent CRT is shown in Table 3 and Fig 4. During the mean follow-up period of 3.8 \pm 3.6 months after undergoing CRT phase image using POOL-SECT showed that all patients had an improvement in their NYHA class to I or II. The LVEF significantly improved $(22.1 \pm 3.7 \%$ vs $36.6 \pm$ $9 \%$ ), but there was no significant reduction in LV end-diastolic diameter $(62 \pm 11 \mathrm{~mm}$ vs $62 \pm 10 \mathrm{~mm})$. The interventricular (average 47 degrees vs 9 degrees) and intraventricular (average 103 degrees vs 67 degrees) asynchrony significantly improved after CRT. The degrees of both inter- and intra-ventricular asynchrony were related to the degree of 
Table 3 Correlations Between Clinical Findings and EF or Ventricular Asynchrony Before and After CRT in 7 Patients

\begin{tabular}{|c|c|c|c|c|c|c|c|c|c|c|}
\hline Disease & $\begin{array}{l}\text { Age } \\
\text { (years) }\end{array}$ & $\begin{array}{c}Q R S \\
\text { complex }\end{array}$ & $\begin{array}{c}E F \\
\text { (pre) } \%\end{array}$ & $\begin{array}{c}E F \\
(\text { post }) \%\end{array}$ & $\begin{array}{l}R V-L V \\
\text { (pre) }\end{array}$ & $\begin{array}{l}R V-L V \\
\text { (post) }\end{array}$ & $\begin{array}{c}S D \text { of mean } \\
\text { phase in } R V \text { (pre) }\end{array}$ & $\begin{array}{c}\text { SD of mean } \\
\text { phase in LV (pre) }\end{array}$ & $\begin{array}{c}\text { SD of mean } \\
\text { phase in } R V \text { (post) }\end{array}$ & $\begin{array}{c}S D \text { of mean } \\
\text { phase in } L V \text { (post) }\end{array}$ \\
\hline$I C M$ & 61 & $V V I$ & 23 & 41 & 33.6 & 4.1 & 108.7 & 94.9 & 97.4 & 70.1 \\
\hline$D C M$ & 76 & $\angle B B B$ & 16 & 21 & 73.7 & 8.2 & 101 & 115.5 & 102 & 79.2 \\
\hline$D C M$ & 31 & Intravent & 19 & 30 & 75.9 & 19.3 & 110 & 101.2 & 64.9 & 67.3 \\
\hline$D C M$ & 76 & $L B B B$ & 25 & 44 & 48 & 6.8 & 118 & 104.7 & 114.8 & 51.2 \\
\hline$D C M$ & 70 & $V V I$ & 21 & 32 & 43.8 & 6.5 & 86.5 & 117.8 & 106 & 73.2 \\
\hline$D C M$ & 57 & $V V I$ & 26 & 43 & 39 & 16.4 & 96.2 & 83.4 & 95.2 & 48.9 \\
\hline$D C M$ & 74 & $L B B B$ & 25 & 45 & 21.4 & 2.9 & 113 & 102.4 & 96 & 80.5 \\
\hline
\end{tabular}

$E F$, ejection fraction; $C R T$, cardiac resynchronization therapy; $R V$, right ventricular; $L V$, left ventricular; $S D$, standard deviation; ICM, ischemic cardiomyopathy; VVI, ventricle $\cdot$ ventricle $\cdot$ inhibit: DCM, dilated cardiomyopathy; LBBB, left bundle branch block.

a

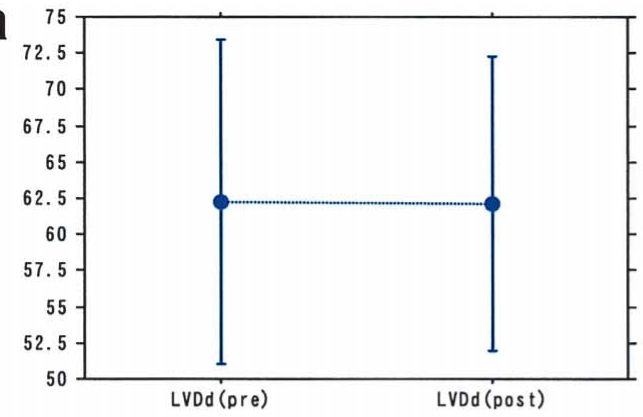

b

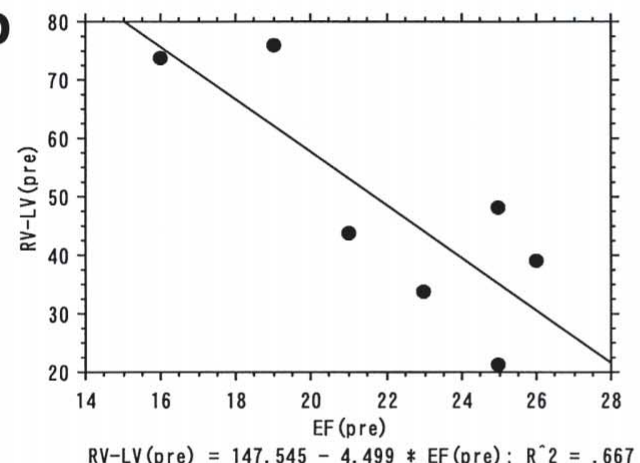

$\mathrm{RV}-\mathrm{LV}(\mathrm{pre})=147.545-4.499 * \mathrm{EF}(\mathrm{pre}) ; \mathrm{R}^{\wedge} 2=.667$

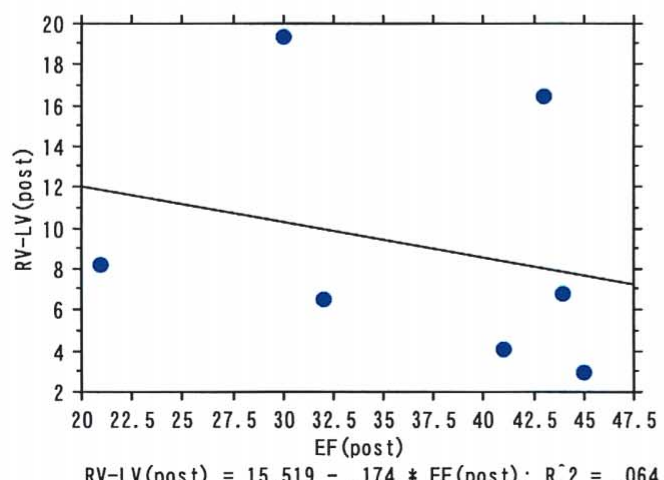

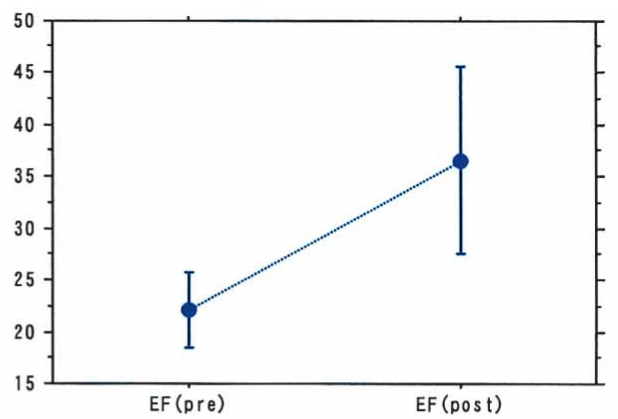

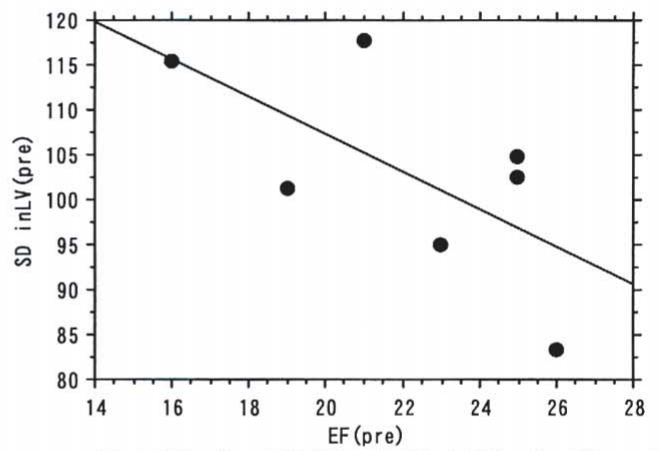

SD inLV (pre) $=148.697-2.071 * E F(p r e) ; R^{\wedge} 2=.417$

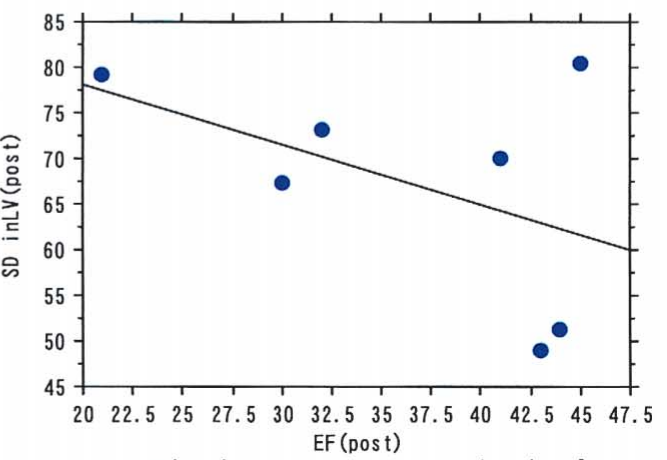

SD inLV(post) $=91.385-.661 * E F($ post $) ; R^{-2} 2=.226$

Fig 4. Correlations between hemodynamic status and ventricular asynchrony before and after cardiac resynchronization therapy (CRT) in 7 patients. (a) Left ventricular end-diastolic diameter and ejection fraction (EF) before and after CRT. (b) Correlations between EF and interventricular asynchrony before and after CRT. (c) Correlations between EF and intraventricular asynchrony before and after CRT. LVDd, left ventricular end-diastolic dimension; RV, right ventricular.

cardiac depression pre CRT (Fig 4). After improvement of these parameters, the remaining inter- or intraventricular asynchrony was not related to the degree of ejection fraction (EF) (Fig 4). One patient died of heart failure 3 years after CRT setting and another died of a non-cardiac event 1 year after CRT setting.

\section{Discussion}

The results of this study confirm the clinical usefulness 
of the phase images obtained by POOL-SPECT for assessing the impact of CRT on LV function. Previous studies used phase analysis to detect mainly the site of accessory conduction pathways and the region of infarction 4,5 Recently it was reported that in approximately $30 \%$ of heart failure patients is associated with intraventricular conduction delay. CRT, which co-ordinates right and LV contraction, has become an established therapy for advanced heart failure.6.7 Although current methods of imaging ventricular asynchrony use Doppler-based approaches, such as Doppler tissue imaging, 8 it was recently shown that the phase images of multigated equilibrium blood pool scintigraphy are useful for evaluating asynchrony. Kerwin et al have reported the efficacy of CRT for improving interventricular asynchrony according to the phase images in conventional 2-dimensional views using multigated equilibrium blood pool scintigraphy $!^{0}$ Fauchier et al have shown that the prognosis of patients with DCM is related to intraventricular rather than inter-ventricular dysynchrony! 1

In our study, the phase images from POOL-SPECT enabled us to easily visualize inter- and intraventricular asynchrony. The patients with both tended to have more deteriorated cardiac function, and experience more fatal cardiac events (Table2). The prognosis of patients with both inter- and intraventricular asynchrony evaluated with phase imaging using POOL-SPECT is worse.

Seven patients in this study were responders for CRT setting in the follow-up by phase imaging using POOLSPECT. There was a significant difference in EF before and after CRT; however Tada et al have reported that there was not a significant difference in LV diameter and EF before and after CRT, and the difference in these results may relate to the observation period. They performed the measurement within 1 month after CRT, whereas our observation period was from within 1 month to 11 months. One evaluated the effect of CRT both short- and long-term, the other evaluated the effect of CRT in the short term. On the phase images, the degrees of both inter- and intraventricular asynchrony were related to the degree of cardiac depression before CRT. Furthermore, the phase images could evaluate the significant improvement in both interand intraventricular asynchrony after CRT.

However, compared with Doppler tissue imaging, phase image using POOL-SPECT for CRT has the disadvantages of cost competitiveness and repetitive technique. However, the efficacy of equilibrium radionuclide-gated blood pool angiography for evaluating the details of cardiac function in the patient with advanced heart failure is commonly recognized and in such cases, it is very useful to be able to evaluate cardiac function, LV volume and the ventricular asynchrony in a single test.

\section{Study Limitations}

To better clarify the efficacy of the phase images of POOL-SPECT, a quantitative assessment is mandatory, although there are many potential difficulties to quantify. For example, although we chose to use the horizontal long-axis view, there are many slices generated in POOL-SPECT. However, decide that this projection was the most valuable because many of the CRT leads that lead to resynchronize are located in the posterolateral wall. More discussion is needed about which projections and slices are better for quantitative measurement. Furthermore, we defined although intraventricular contractile asynchrony as the SD of the mean phase angle for the LV and RV blood pools, we are concerned about the possibility that the width of phase distribution might be corrected by the size of the ventricle. More clinical cases and foundamental experiments are needed.

\section{Conclusion}

The phase images of POOL-SPECT are useful for assessing inter- and intraventricular asynchrony, and the technique has potential for predicting the indication for CRT in patients with advanced heart failure.

\section{References}

1. David JB, Elizabeth AB, Kenneth LB, Ronald DB, Hugh C, Steven $\mathrm{NG}$, et al. Cardiac resynchronization and death from progressive heart failure. JAMA 2003; 289: 730-740.

2. Abraham WT. Rationale and design of a randomized clinical trial to assess the safety and efficacy of cardiac resynchronization therapy in patients with advanced heart failure: The Multicenter InSync Randomized Clinical Evaluation (MIRACLE). J Card Fail 2000; 6: 369-380.

3. Higgins SL, Hummel JD, Niazi IK, Giudici MC, Worley SJ, Saxon LA, et al. Cardiac resynchronization therapy for the treatment of heart failure in patients with intraventricular conduction delay and malignant ventricular tachyarrhythmias. J Am Coll Cardiol 2003; 42: $1454-1459$.

4. Nakajima K, Bunko H, Tada A, Tonami N, Hisada K, Misaki T, et al. Nuclear tomographic phase analysis: Localization of accessory conduction pathway in patients with Wolff-Parkinson-White syndrome. Am Heart J 1985; 109: 809-815.

5. Nakajima K, Bunko H, Tonami N, Taki J, Nanbu I, Shiire Y, et al. Length-based Fourier analysis in the pre-excitation syndrome. J Nucl Med 1986; 27: 1131-1137.

6. Farwell D, Patel NR, Hall A, Ralph S, Sulke AN. How many people with heart failure are appropriate for biventricular resynchronization? Eur Heart J 2000; 21: 1246-1250.

7. Patel AR, Konstam MA. Recent advances in the treatment of heart failure. Circ J 2002; 66: 117-121.

8. Sogaard P, Kim WY, Jensen HK, Mortensen P, Pedersen AK, Kristensen $\mathrm{BO}$, et al. Impact of acute biventricular pacing on left ventrivular performance and volumes in patients with severe heart failure: A tissue Doppler and three-dimensional echocardiographic study. Cardiology 2001; 95: 173-182.

9. Toda H, Toide H, Naito S, Kurosaki K, Ito S, Miyaji K, et al. Tissue Doppler imaging and strain Doppler imaging as modalities for predicting clinical improvement in patients receiving biventricular pacing. Circ J 2005; 69: 194-200.

10. Kerwin WF, Botvinick EH, O'Connell JW, Merrick SH, DeMarco T, Chatterjee $\mathrm{K}$, et al. Ventricular contraction abnormalities in dilated cardiomyopathy: Effect of biventricular pacing to correct interventricular dyssynchrony. J Am Coll Cardiol 2000; 35: 1221 - 1227.

11. Fauchier L, Marie O, Casset-Senon D, Babuty D, Cosnay P, Fauchier JP. Interventricular and intraventricular dyssynchrony in idiopathic dilated cardiomyopathy. J Am Coll Cardiol 2002; 40: 2022-2030. 